\section{Illumination: Inferred or observed?}

\author{
HOWARD R. FLOCK \\ York University, Downsview, Ontario, Canada
}

In perception, a direct correspondence exists between what we see and what is out there. When we see a tree, it is because there is a tree out there and our gaze is directed toward it. When we direct our gaze away from the tree, it disappears from sight. We no longer see it. Correspondingly, the gaze may be directed toward a source of light, in which case we see the light source. When we direct our gaze away from the light source, it too disappears from sight-or does it?

Some theoretical explanations of why we see achromatic colors (blacks, grays, and whites) require information about the illuminating light source. When the source is not visible, as, for example, in strolling among the canyons of New York City, that becomes a problem for those theories. With the source not visible, various kinds of external cues and clues to the illumination have been hypothesized. Also, based on prior experiences, the perceiver may have an internal representation of what the illumination is. These external indicators and internal representations are utilized by the visual/ perceptual processor, in order to infer the illumination and form achromatic perceptions. In support of such theories, research has attempted with only mixed success to involve such indicators of the illumination in the perception of achromatic color.

Actually, the visual stimulus for real-world scenes is redundant with direct images of the illuminating sources. The surfaces of the real world vary from being very matte to very specular. Every surface is to some degree matte as well as specular, as Hunter (1975) has pointed out. Moreover, the surfaces of the real world are complexly curved and oriented in every direction, not planar in just one direction. As a result of this complex curvature in specular surfaces, the eye of a perceiver is likely to pick up specular images as he scans real-world spaces.

A perfect specular surface is a perfect mirror. A mirror images the world that it "sees," including any illuminating sources. The mirror produces what is called a virtual image of its surrounds. As a mirror is made progressively less perfect, images become less distinct, then more blurred, degraded, and finally indistinguishable from the surrounds. Even with considerable degradation of mirror images, it is possible to detect characteristics of virtual images, as all of us can demonstrate by projecting transparencies and then slowly defocusing them. At an extreme, the mirror images will be totally degraded: images and the light producing them will be homogenized as a uniformly diffused area.

The author's mailing address is: Department of Psychology, York University, Downsview, Ont., Canada M3J 1 P3.
The real world of objects and surfaces may be thought of as a giant specular mirror of varying quality. At one extreme is the placid surface of a body of water, mirroring the scene with uncanny realism. At the other extreme is the "zero" mirror of dry, dusty dirt on a summer day. In between are surfaces with varying degrees of resemblance to a mirror, such as facets of rock that have been polished by wind and water; the glossy surfaces of summer leaves; the sheen of hair; a field of summer grass, and so on. Many of man's artifacts tend to be very good mirrors. Examples abound, as in the case of automobile bodies, telephone casings, fittings, filing cabinets, porcelains, glazes, plastics, enamel and semienamel paints, polished furniture, and fabrics such as leather. Whenever man (or nature) continuously polishes a surface, the surface becomes transformed into a better and better mirror.

Even surfaces that are generally thought of as being highly matte, not specular at all, are not totally devoid of specularity. As an example, place a point source of light nearly edge-on to a piece of mimeograph paper that is placed flat on a table. Next, look at the surface from nearly edge-on, but from the opposite edge. You will see a highly degraded image of the light source.

The real world is populated, as you see, by "mirrors" of varying quality, from very good ones to hopelessly bad ones. Because most natural surfaces are rarely flat, the virtual images of these illuminating sources will be visible to the observer, regardless of his viewing position. Vision, therefore, is redundantly supplied with direct images of sources. With such an abundant supply, would the visual system really need the indirect information about illumination, which has been hypothesized to derive from cues, clues, internal representations, and schema?

Many theories of achromatic perception use an algorithm that requires illumination to be registered, perceived, or apprehended at some level of the visual process. These theories have generated illumination as an inferred construct. That is no longer necessary. Revision of such theory could substitute direct images of the illuminating source. It would then be in order to ask: how does the visual system use these specular "mirror" images of illuminating sources? That is an empirical question, and it should be addressed. [Virtually no experimenters have investigated the effects of specularity on achromatic perceptions. An outstanding exception to this is Beck (1964). His paper is truly a pioneering work.]

\section{REFERENCES}

Beck, J. (1964). The effect of gloss on perceived lightness. American Journal of Psychology, 77, 54-63.

Hunter, R. S. (1975). The measurement of appearance. New York: Wiley.

(Manuscript received November 18, 1983; accepted for publication January 12, 1984.) 